K. Shiraiwa and M. Kurata

Nagoya Math. J.

Vol. 82 (1981), 83-97

\title{
A GENERALIZATION OF A THEOREM OF MAROTTO
}

\author{
KENICHI SHIRAIWA AND MASAHIRO KURATA
}

In 1975, $\mathrm{Li}$ and Yorke [3] found the following fact. Let $f: I \rightarrow I$ be a continuous map of the compact interval $I$ of the real line $R$ into itself. If $f$ has a periodic point of minimal period three, then $f$ exhibits chaotic behavior. The above result is generalized by F.R. Marotto [4] in 1978 for the multi-dimensional case as follows. Let $f: \boldsymbol{R}^{n} \rightarrow \boldsymbol{R}^{n}$ be a differentiable map of the $n$-dimensional Euclidean space $\boldsymbol{R}^{n}(n \geqq 1)$ into itself. If $f$ has a snap-back repeller, then $f$ exhibits chaotic behavior.

In this paper, we give a generalization of the above theorem of Marotto. Our theorem can also be regarded as a generalization of the Smale's results [6] on the transversal homoclinic point of a diffeomorphism.

\section{§1. The Main Theorem}

Let $M$ be a smooth manifold of dimension $n$. We denote by $T_{x}(M)$ the tangent space of $M$ at a point $x$ of $M$. Let $f: M \rightarrow M$ be a $C^{1}$-map. The tangent map of $f$ at $x \in M$ is denoted by $T_{x} f: T_{x}(M) \rightarrow T_{f(x)}(M)$.

Let $z_{0} \in M$ be a fixed point of $f$. Then $f$ is called a hyperbolic fixed point if all the modulus of the eigenvalues of $T_{z_{0}} f: T_{z_{0}}(M) \rightarrow T_{z_{0}}(M)$ are different from 0 and 1 . Define $E^{s}$ (resp. $E^{u}$ ) to be the direct sum of the generalized eigenspaces of $T_{z_{0}} f$ which correspond to the eigenvalues of modulus less than 1 (resp. greater than 1). Then $E^{s}$ and $E^{u}$ are $T_{z_{0}} f$ invariant vector subspaces of $T_{z_{0}}(M)$, and $T_{z_{0}}(M)=E^{s} \oplus E^{u}$.

Let $s=\operatorname{dim} E^{s}$ and $u=\operatorname{dim} E^{u}$. Fix a norm $\|\cdot\|$ on $E^{s}$ and $E^{u}$. For $r_{0}>0$, we define $E^{s}\left(r_{0}\right)=\left\{x \in E^{s} ;\|x\| \leqq r_{0}\right\}$ and $E^{u}\left(r_{0}\right)=\left\{x \in E^{u} ;\|x\| \leqq r_{0}\right\}$. By stable manifold theorem, it is known that there are embeddings $\varphi^{s}$ : $E^{s}\left(r_{0}\right) \rightarrow M$ and $\varphi^{u}: E^{u}\left(r_{0}\right) \rightarrow M$ for sufficiently small $r_{0}>0$ satisfying the following conditions.

(1) $\varphi^{s}(0)=z_{0}$ and $\varphi^{u}(0)=z_{0}$

(2) $T_{0} \varphi^{s}: T_{0}\left(E^{s}\left(r_{0}\right)\right)=E^{s} \rightarrow T_{z_{0}}(M)$ and $T_{0} \varphi^{u}: T_{0}\left(E^{u}\left(r_{0}\right)\right)=E^{u} \rightarrow T_{z_{0}}(M)$ are injections.

Received July 3, 1979. 
(3) If we put $W_{\mathrm{loc}}^{s}\left(z_{0}\right)=\varphi^{s}\left(E^{s}\left(r_{0}\right)\right)$ and $W_{\mathrm{loc}}^{u}\left(z_{0}\right)=\varphi^{u}\left(E^{u}\left(r_{0}\right)\right)$, then there exists a neighborhood $U$ of $z_{0}$ such that $W_{\mathrm{loc}}^{s}\left(z_{0}\right)=\left\{x \in U ; \lim _{n \rightarrow \infty} f^{n}(x)=\right.$ $\left.z_{0}\right\}$ and $W_{\mathrm{loc}}^{u}\left(z_{0}\right)=\left\{x \in U ; \lim _{n \rightarrow \infty} f^{-n}(x)=z_{0}\right\}$.

We call such $W_{\text {loc }}^{s}\left(z_{0}\right)$ (resp. $W_{\text {loc }}^{u}\left(z_{0}\right)$ ) a local stable (resp. unstable) manifold of $f$ at $z_{0}$.

Now we fix a metric $d$ on $M$.

MaIn Theorem. Let $f: M \rightarrow M$ be a $C^{1}$-map. Let $z_{0} \in M$ be a hyperbolic fixed point of $f$. Now we assume the following three conditions.

(1) $u=\operatorname{dim} E^{u}>0$.

(2) There exist a point $z_{1} \in W_{\mathrm{loc}}^{u}\left(z_{0}\right)\left(z_{1} \neq z_{0}\right)$ and a positive integer $m$ such that $f^{m}\left(z_{1}\right) \in W_{\text {loc }}^{s}\left(z_{0}\right)$.

(3) There exists a u-dimensional disk $B^{u}$ embedded in $W_{\mathrm{loc}}^{u}\left(z_{0}\right)$ such that $B^{u}$ is a neighborhood of $z_{1}$ in $W_{\mathrm{loc}}^{u}\left(z_{0}\right), f^{m} \mid B^{u}: B^{u} \rightarrow M$ is an embedding, and $f^{m}\left(B^{u}\right)$ intersects $W_{\mathrm{loc}}^{s}\left(z_{0}\right)$ transversally at $f^{m}\left(z_{1}\right)$.

Then the following conclusion holds.

(a) There is a positive integer $N$ such that there is a periodic point of $f$ of minimal period $p$ for any integer $p \geqq N$.

(b) There is an uncountable set $S$ (called a scrambled set) in $M$ satisfying the following conditions.

(i) $S$ does not contain any periodic points.

(ii) $f(S) \subset S$

(iii) $\lim \sup _{k \rightarrow \infty} d\left(f^{k}(x), f^{k}(y)\right)>0$ for any $x, y \in S(x \neq y)$.

(iv) $\lim \sup _{k \rightarrow \infty} d\left(f^{k}(x), f^{k}(y)\right)>0$ for any $x \in S$ and a periodic point $y$.

(v) There is an uncountable subset $S_{0}$ contained in $S$ such that

$$
\lim \inf _{k \rightarrow \infty} d\left(f^{k}(x), f^{k}(y)\right)=0 \quad \text { for any } x, y \in S_{0} \text {. }
$$

Remark 1. The above theorem holds if $f: M \rightarrow M$ is of class $C^{1}$ on a neighborhood of $z_{0}$ and on a neighborhood of the orbit of $z_{1}$.

Remark 2. In the above theorem $T_{z_{1}} f^{m}$ may be degenerate.

Remark 3. In case $u=\operatorname{dim} M$ and $f^{m}\left(z_{1}\right)=z_{0}$, the above theorem reduces to the theorem of Marotto.

Remark 4. If $f$ is a diffeomorphim with $f^{m}\left(z_{1}\right) \neq z_{0}$, then the above assumption implies that $f^{m}\left(z_{1}\right)$ is a transversal homoclinic point.

Remark 5. Transversality condition in our assumption (3) is necessary. We have an example which shows that without the transversality 
condition the conclusion of the main theorem does not hold.

Throughout this paper, we work under the assumption of the main theorem and use the same notation.

\section{§2. Main Lemma}

Using the inverse function theorem, we know that there is an embedding $\Phi: E^{s}\left(r_{1}\right) \times E^{u}\left(r_{1}\right) \rightarrow M$ such that $\Phi \mid E^{s}\left(r_{1}\right) \times 0=\varphi^{s}$ and $\Phi \mid 0 \times E^{u}\left(r_{1}\right)$ $=\varphi^{u}$ for sufficiently small $r_{1}>0$. Therefore, we identify $E^{s}\left(r_{1}\right) \times E^{u}\left(r_{1}\right)$ with a neighborhood of $z_{0}$ for sufficiently small $r_{1}$. By this identification, $E^{s}\left(r_{1}\right)$ (resp. $\left.E^{u}\left(r_{1}\right)\right)$ is identified with $W_{\mathrm{loc}}^{s}\left(z_{0}\right)$ (resp. $W_{\mathrm{loc}}^{u}\left(z_{0}\right)$ ).

Since $z_{0}$ is a hyperbolic fixed point of $f$ by our assumption, $T_{z_{0}} f$ is non-degenerate. Also, we assume that $T_{x} f$ is non-degenerate for any point $x \in E^{s}\left(r_{1}\right) \times E^{u}\left(r_{1}\right)$ (We replace $r_{1}>0$ a smaller value if necessary).

For $x \in E^{s}\left(r_{1}\right) \times E^{u}\left(r_{1}\right)$, we write

$$
T_{x} f=\left(\begin{array}{cc}
T_{x}^{s} & A_{x} \\
B_{x} & T_{x}^{u}
\end{array}\right), \quad T_{x} f^{-1}=\left(\begin{array}{cc}
T_{x}^{\prime s} & A_{x}^{\prime} \\
B_{x}^{\prime} & T_{x}^{\prime u}
\end{array}\right)
$$

with respect to the product structure $E^{s}\left(r_{1}\right) \times E^{u}\left(r_{1}\right)$.

Put

$$
\begin{aligned}
a & =\max _{x}\left\{\left\|T_{x}^{s}\right\|,\left\|T_{x}^{\prime u}\right\|\right\}, \\
\frac{1}{b} & =\max _{x}\left\{\left\|T_{x}^{u-1}\right\|,\left\|T_{x}^{s^{-1}}\right\|\right\},
\end{aligned}
$$

and

$$
k=\max _{x}\left\{\|\left\{A_{x}\|,\| B_{x}\|,\| A_{x}^{\prime}\|,\| B_{x}^{\prime} \|\right\}\right\},
$$

where $x$ runs over $E^{s}\left(r_{1}\right) \times E^{u}\left(r_{1}\right)$. Then, by our hyperbolicity assumption on $z_{0}$, the following inequalities hold on a sufficiently small neighborhood $E^{s}\left(r_{1}\right) \times E^{u}\left(r_{1}\right)$.

(*)

$$
\left(\begin{array}{l}
a<1, \quad \frac{1}{b}<1, \\
a+k<1, \quad b-k>1, \quad \text { and } \quad k<\frac{(b-1)^{2}}{4}
\end{array}\right.
$$

Since $z_{1} \in W_{\mathrm{loc}}^{u}\left(z_{0}\right)$ by our assumption, we may assume that $z_{1} \in E^{u}\left(r_{1}\right)$. Choose a real number $r$ such that $0<r<r_{1}$ and $z_{1} \notin E^{u}(r)$.

For $\sigma=s, u, \pi^{\sigma}$ denotes the natural projection from $E^{s}\left(r_{1}\right) \times E^{u}\left(r_{1}\right)$ 
onto $E^{o}\left(r_{1}\right)$. Since $T_{x}\left(E^{s}\left(r_{1}\right) \times E^{u}\left(r_{1}\right)\right)=T_{x}\left(E^{s}\left(r_{1}\right)\right) \times T_{x}\left(E^{u}\left(r_{1}\right)\right)=E^{s} \times E^{u}$, $v \in T_{x}\left(E^{s}\left(r_{1}\right) \times E^{u}\left(r_{1}\right)\right)$ is uniquely expressed as $v=v^{s}+v^{u}, v^{\sigma} \in E^{o}$.

For a $\sigma$-dimensional disk $D^{\sigma}$ in $E^{\sigma}\left(r_{1}\right)$, we denote its boundary in $E^{\sigma}\left(r_{1}\right)$ by $\partial D^{\sigma}$.

MaIn Lemma. Assume the same conditions of the main theorem. Let $B^{u}$ be an u-dimensional disk in $E^{u}\left(r_{1}\right)$, and let $B^{s}$ be an arbitrary s-dimensional disk with the origin 0. If $\psi: B^{s} \times B^{u} \rightarrow E^{s}\left(r_{1}\right) \times E^{u}\left(r_{1}\right)$ is an embedding such that $\psi \mid 0 \times B^{u}$ is the inclusion map $B^{u} \subset E^{u}\left(r_{1}\right)$, then for any $\varepsilon$ $>0$ and $L>0$ there exists a positive integer $N(\psi, \varepsilon, L)$ satisfying the following conditions.

For any integer $n \geqq N(\psi, \varepsilon, L)$, there is an embedding $\phi=\phi(\psi, \varepsilon, L, n)$ : $E^{s}(r) \times B^{u} \rightarrow E^{s}\left(r_{1}\right) \times E^{u}\left(r_{1}\right)$ satisfying the following eight conditions.

$$
\begin{aligned}
& \phi\left(E^{s}(r) \times y\right) \subset \psi\left(B^{s} \times y\right) \quad \text { for } y \in B^{u} . \\
& f^{-n}\left(\phi\left(E^{s}(r) \times B^{u}\right)\right) \subset E^{s}(r) \times E^{u}(r) . \\
& f^{-n}\left(\phi\left(\partial E^{s}(r) \times B^{u}\right)\right) \subset \partial E^{s}(r) \times E^{u}(r) . \\
& \pi^{s} f^{-n} \phi\left(x \times B^{u}\right)=x \text { for } x \in E^{s}(r) \text {. } \\
& \left\|v^{u}\right\|<\varepsilon\left\|v^{s}\right\| \text { for any non-zero } v \text { in } T\left(f^{-n} \phi\left(E^{s}(r) \times y\right)\right), y \in B^{u} . \\
& \left\|\left(T f^{-n}(v)\right)^{s}\right\|>L\left\|v^{s}\right\| \text { for any non-zero } v \text { in } T\left(\phi\left(E^{s}(r) \times y\right)\right), y \in B^{u} . \\
& \left\|\left(T f^{n}(v)\right)^{s}\right\|<\varepsilon\left\|\left(T f^{n}(v)\right)^{u}\right\| \text { and } \\
& \left\|\left(T f^{n}(v)\right)^{u}\right\|>L\left\|v^{u}\right\| \text { for any non-zero } v \text { in } T\left(f^{-n} \phi\left(x \times B^{u}\right)\right), x \in E^{s}(r) .
\end{aligned}
$$

Proof. Let $r_{2}$ and $\varepsilon_{1}$ be real numbers such that $r<r_{2}<r_{1}$ and $0<\varepsilon_{1}$ $<\min \left\{\varepsilon, r, r_{2}-r, r_{1}-r_{2}\right\}$. Since the set $\left\{\left\|v^{u}\right\| /\left\|v^{s}\right\| ; v \neq 0 \in T_{y}\left(\psi\left(B^{s} \times y\right)\right)\right.$, $\left.y \in B^{u}\right\}$ is bounded, there exists an integer $N_{1}>0$ such that some $s$-dimensional disk in $f^{-n} \psi\left(B^{s} \times y\right)$ is $\varepsilon_{1} C^{1}$-close to $E^{s}\left(r_{2}\right)$ for any $n \geqq N_{1}$ and $y \in$ $B^{u}$. This is proved by the same argument as in the proof of the $\lambda$-lemma in J. Palis [5], if we note that the $\lambda$-lemma holds uniformly with respect to a disk family $\left\{\psi\left(B^{s} \times y\right) ; y \in B^{u}\right\}$.

The above fact implies that there exists a neighborhood $V$ of $B^{u}$ in $B^{s} \times B^{u}$ such that $f^{-n}\left(\psi\left(\left(B^{s} \times y\right) \cap V\right)\right)$ is an $s$-dimensional disk, which is $\varepsilon_{1} C^{1}$-close to $E^{s}\left(r_{1}\right)$ and $\pi^{s} f^{-n}\left(\psi\left(\left(B^{s} \times y\right) \cap V\right)\right) \supset E^{s}(r)$ for any $y \in B^{u}$. If we take $\varepsilon_{1}>0$ small enough, then $f^{-n}\left(\psi\left(\left(B^{s} \times y\right) \cap V\right)\right)$ intersects with $x \times E^{u}(r)$ $\left(x \in E^{s}(r)\right)$ in a single point for each $y \in B^{u}$. We denote this point by $\chi(x, y)$. Now, define $\phi: E^{s}(r) \times B^{u} \rightarrow E^{s}\left(r_{1}\right) \times E^{u}\left(r_{1}\right)$ by 


$$
\phi(x, y)=f^{n}(\chi(x, y)) \quad \text { for }(x, y) \in E^{s}(r) \times B^{u} .
$$

Then, the conditions $(2.1) \sim(2.5)$ are clearly satisfied by the definition of $\phi$.

If $N_{1}$ is large enough, then there is a neighborhood $W$ of $B^{u}$ in $\psi\left(B^{s} \times\right.$ $\left.B^{u}\right)$ such that for any non-zero $v$ in $T_{z}\left(\psi\left(B^{s} \times y\right)\right)$ with $y \in B^{u}$ and $z \in W$ $\cap \psi\left(B^{s} \times y\right)$ the inequality

$$
\left\|\left(T f^{-N_{1}}(v)\right)^{u}\right\| /\left\|\left(T f^{-N_{1}}(v)\right)^{s}\right\|<1
$$

holds. Then,

$$
\begin{aligned}
\left\|\left(T f^{-N_{1}-1}(v)\right)^{s}\right\| & \geqq\left\|\left(T^{\prime s}\right)^{-1}\right\|^{-1} \cdot\left\|\left(T f^{-N_{1}}(v)\right)^{s}\right\|-\left\|A^{\prime}\right\|\left\|\left(T f^{-N_{1}}(v)\right)^{u}\right\| \\
& >(b-k)\left\|\left(T f^{-N_{1}}(v)\right)^{s}\right\|
\end{aligned}
$$

and

$$
\begin{aligned}
\frac{\left\|\left(T f^{-N_{1}-1}(v)\right)^{u}\right\|}{\left\|\left(T f^{-N_{1}-1}(v)\right)^{s}\right\|} & <\frac{\left\|T^{\prime u}\right\|\left\|\left(T f^{-N_{1}}(v)\right)^{u}\right\|+\left\|B^{\prime}\right\|\left\|\left(T f^{-N_{1}}(v)\right)^{s}\right\|}{(b-k)\left\|\left(T f^{-N_{1}}(v)\right)^{s}\right\|} \\
& \leqq(b-k)^{-1}\left(a \frac{\left\|\left(T f^{-N_{1}}(v)\right)^{u}\right\|}{\left\|\left(T f^{-N_{1}}(v)\right)^{s}\right\|}+k\right) \\
& \leqq(b-k)^{-1}(a+k)<1 .
\end{aligned}
$$

The last inequality holds since $b-k>1, a+k<1$.

By the induction on $\ell \geqq 1$, we have the following inequality

$$
\left\|\left(T f^{-N_{1}-\ell}(v)\right)^{s}\right\|>(b-k)^{\ell}\left\|\left(T f^{-N_{1}-\ell}(v)\right)^{u}\right\|
$$

for any $\ell \geqq 1$ and any non-zero $v \in T_{z}\left(\psi\left(B^{s} \times y\right)\right)$, where $y \in B^{u}, z \in W \cap$ $\psi\left(B^{u} \times y\right)$.

Now taking $N>N_{1}$ large enough, we have (2.6) for any $n \geqq N$.

Replacing $T f^{-1}$ (resp. $E^{u}$ and $E^{s}$ ) by $T f$ (resp. $E^{s}$ and $E^{u}$ ), we have (2.7) and (2.8) similarly.

\section{§3. Construction of a map from a shift}

First, we construct symbols, and using these symbols, we define a map from a shift to $M$. This map is used in our proof of the main theorem.

Lemma 1. There is a positive integer $N_{1}$ such that for any integer $N_{0}$ $\geqq N_{1}$, there are two embeddings

$$
\phi_{i}:\left(E^{s}(r) \times E^{u}\left(r_{2}\right), E^{s}(r) \times B_{i}^{u}\right) \longrightarrow E^{s}\left(r_{2}\right) \times E^{u}\left(r_{2}\right) \quad(i=0,1)
$$

$\left(0<r<r_{2}<r_{1}\right)$ of a pair of rectangles, where $B_{i}^{u}$ is a u-dimensional disk contained in the interior of $E^{u}\left(r_{2}\right)$, satisfying the following 11 conditions. 
$\left(f^{N_{i}}\right)_{*}: H_{u-1}\left(\phi_{i}\left(E^{s}(r) \times \partial B_{i}^{u}\right)\right) \rightarrow H_{u-1}\left(\phi_{j}\left(E^{s}(r) \times\left(E^{u}\left(r_{2}\right)-B_{j}^{u}\right)\right)\right)$ is an isomorphism, where $H_{u-1}(\quad)$ is the $(u-1)$-th homology group and $\left(f^{N_{i}}\right)_{*}$ is the induced homomorphism of $f^{N_{i}}(i, j=0,1)$.

(3.4) $\pi^{s} \phi_{i}\left(x \times B_{i}^{u}\right)$ consists of a single point for $x \in E^{s}(r)$, and $\pi^{s} \phi_{i}\left(E^{s}(r) \times B_{i}^{u}\right)$ $=E^{s}(r) \quad(i=0,1)$.

(3.5) $\quad \pi^{u} f^{N_{i}} \phi_{i}\left(E^{s}(r) \times y\right)$ consists of a single point for $y \in B_{i}^{u} \quad(i=0,1)$.

(3.6) $2\left\|v^{s}\right\|<\left\|v^{u}\right\|$ for any non-zero $v$ in $T\left(f^{N_{i}} \phi_{i}\left(x \times B_{i}^{u}\right)\right), x \in E^{s}(r)$

$$
(i=0,1) \text {. }
$$

(3.7) $2\left\|v^{u}\right\|<\left\|v^{s}\right\|$ for any non-zero $v$ in $T\left(\phi_{i}\left(E^{s}(r) \times y\right)\right), y \in B_{i}^{u}$

$$
(i=0,1) \text {. }
$$

(3.8) $\left\|\left(T f^{N_{i}}(v)\right)^{u}\right\|>8\left\|v^{u}\right\|$ for any non-zero $v$ in $T\left(\phi_{i}\left(x \times B_{i}^{u}\right)\right), x \in E^{s}(r)$

$$
(i=0,1) \text {. }
$$

(3.9) $8\left\|\left(T f^{N_{i}}(v)\right)^{s}\right\|<\left\|v^{s}\right\|$ for any non-zero $v$ in $T\left(\phi_{i}\left(E^{s}(r) \times y\right)\right), y \in B_{i}^{u}$

$$
(i=0,1) \text {. }
$$

(3.10) If we put $A_{0}=\phi_{0}\left(E^{s}(r) \times B_{0}^{u}\right)$ and $A_{1}=\phi_{1}\left(E^{s}(r) \times B_{1}^{u}\right)$,

$$
\text { then } A_{0} \cap A_{1}=\phi \text {. }
$$

(3.11) There exists an integer $k\left(0 \leqq k \leqq N_{0}-1\right)$ such that $f^{k}\left(A_{1}\right) \cap f^{i}\left(A_{0}\right)$ $=\phi$ for $0 \leqq i \leqq N_{0}-1$ and $f^{k}\left(A_{1}\right) \cap f^{i}\left(A_{1}\right)=\phi$ for $0 \leqq i \neq k \leqq N_{1}-1$.

Proof. Since $z_{1} \in W_{\mathrm{loc}}^{u}\left(z_{0}\right)=E^{u}\left(r_{1}\right)$ and $z_{1} \neq z_{0}$ by our assumption, there exists a positive number $r_{2}$ such that $z_{1} \notin E^{u}\left(r_{2}\right), r_{2}<r_{1}$. Let $r$ be a sufficiently small number such that $0<r<r_{2}$, which we determine later.

Since $f^{-1} \mid E^{u}\left(r_{1}\right)$ is a contraction, there is a positive integer $L$ such that $f^{-L}\left(z_{1}\right) \in E^{u}\left(r_{2}\right)$.

Let $j: E^{s}(r) \times E^{u}(r) \rightarrow E^{s}\left(r_{2}\right) \times E^{u}\left(r_{2}\right)$ be the inclusion map. Let $N_{2}=$ $N(j, 1 / 2,8)$ be the integer given in the main lemma. By the $\lambda$-lemma, the transversality condition of the main theorem, and the fact that $f \mid E^{u}\left(r_{1}\right)$ is an expansion, there are positive number $N_{3}>N_{2}$ and an $u$-dimensional disk $D^{u}$ in $E^{u}\left(r_{2}\right)$ satisfying the following conditions.

(1) $D^{u}$ is a neighborhood of $f^{-L}\left(z_{1}\right)$ in $E^{u}\left(r_{2}\right)$.

(2) $f^{N_{s}}\left(f^{-L}\left(z_{1}\right)\right) \in E^{s}(r)$ and $f^{N_{s}}\left(D^{u}\right) \subset E^{s}(r) \times E^{u}\left(r_{2}\right)$. 
(3) $f^{N_{3}}\left(\partial D^{u}\right) \subset E^{s}(r) \times\left(E^{u}\left(r_{2}\right)-E^{u}(r)\right)$.

(4) $8\left\|v^{s}\right\|<\left\|v^{u}\right\|$ for any non-zero $v \in T\left(f^{v_{s}}\left(D^{u}\right)\right)$.

(5) $D^{u} \cap E^{u}(r)=\phi$.

(6) There exists a positive number $r_{3}$ such that $f^{L}\left(D^{u}\right) \subset \operatorname{Int} E^{u}\left(r_{3}\right)$ $-f^{-1}\left(E^{u}\left(r_{3}\right)\right)$, and $f^{L+i}\left(D^{u}\right) \cap f^{L}\left(D^{u}\right)=\phi$ for $1 \leqq i \leqq N_{3}-L$.

From conditions (3) and (4), $\pi^{u} \mid f^{N_{3}}\left(D^{u}\right): f^{N_{3}}\left(D^{u}\right) \rightarrow E^{u}\left(r_{2}\right)$ is a diffeomorphism onto its image, and its image contains $E^{u}(r)$. And there is a neighborhood $W$ of $D^{u}$ in $E^{s}\left(r_{2}\right) \times E^{u}\left(r_{2}\right)$ such that

(7) $\pi^{u} \circ\left(f^{N_{s}} \mid W\right)$ is a submersion,

(8) $f^{L}(W) \cap E^{s}(r) \times E^{u}(r)=\phi$,

(9) $f^{L}(W) \subset E^{s}(\delta) \times E^{u}\left(r_{3}\right)-f^{-1}\left(E^{s}(\delta) \times E^{u}\left(r_{3}\right)\right)$ for some $\delta>0$, and

(10) $f^{L+i}(W) \cap f^{L}(W)=\phi$ for $1 \leqq i \leqq N_{3}-L$.

Therefore, there are positive number $\delta^{\prime}$ and an embedding $\psi: E^{s}\left(\delta^{\prime}\right) \times D^{u}$ $\rightarrow E^{s}\left(r_{2}\right) \times E^{u}\left(r_{2}\right)$ satisfying the following conditions.

(11) $\psi \mid D^{u}$ is the inclusion map, and $\psi\left(E^{s}\left(\delta^{\prime}\right) \times D^{u}\right) \subset W$.

(12) $\pi^{u} \circ f^{N_{3}}\left(\psi\left(E^{s}\left(\delta^{\prime}\right) \times y\right)\right)$ consists of a single point for $y \in D^{u}$.

(13) $\pi^{s}\left(\psi\left(x \times D^{u}\right)\right)=x$ for $x \in E^{s}\left(\delta^{\prime}\right)$.

(14) $\psi\left(E^{s}\left(\delta^{\prime}\right) \times D^{u}\right) \cap E^{s}(r) \times E^{u}(r)=\phi$.

If we take $\delta^{\prime}>0$ small enough, then we have the following conditions.

(15) $f^{v_{3}}\left(\psi\left(E^{s}\left(\delta^{\prime}\right) \times D^{u}\right)\right) \subset E^{s}(r) \times E^{u}\left(r_{2}\right)$

(16) $f^{N_{s}}\left(\psi\left(E^{s}\left(\delta^{\prime}\right) \times \partial D^{u}\right)\right) \subset E^{s}(r) \times\left(E^{u}\left(r_{2}\right)-E^{u}(r)\right)$.

(17) $4\left\|v^{s}\right\|<\left\|v^{u}\right\|$ for any non-zero $v$ in $T\left(f^{N_{3}}\left(\psi\left(x \times D^{u}\right)\right)\right), x \in E^{s}\left(\delta^{\prime}\right)$.

By (17) we can take a small number $\rho(0<\rho<1 / 2)$ such that

(18) $2\left\|\left(T f^{N_{s}}(v)\right)^{s}\right\|<\left\|\left(T f^{N_{s}}(v)\right)^{u}\right\|$ if $v \in T\left(\psi\left(E^{s}\left(\delta^{\prime}\right) \times D^{u}\right)\right)$ and $\left\|v^{s}\right\|<\rho\left\|v^{u}\right\|$.

By a similar argument, we can take a large number $L_{1}>0$ such that

(19) $\left\|\left(T f^{N_{3}}(v)\right)^{u}\right\|>L_{1}^{-1}\left\|v^{u}\right\|$ for any non-zero

$v \in T\left(\psi\left(E^{s}\left(\delta^{\prime}\right) \times D^{u}\right)\right.$ with $\left\|v^{s}\right\|<\rho\left\|v^{u}\right\|$, and

(20) $\left\|\left(T f^{N_{3}}(v)\right)^{s}\right\|<L_{1}\left\|v^{s}\right\|$ for any non-zero

$v \in T\left(\psi\left(E^{s}\left(\delta^{\prime}\right) \times y\right)\right), y \in D^{u}$.

Let $N_{4}=N\left(\psi, \rho, 8 L_{1}\right)$ be the positive integer given in the main lemma, and $N_{1}=N_{3}+N_{4}$. Let $\phi_{1}: E^{s}(r) \times D^{u} \rightarrow E^{s}(r) \times E^{u}(r)$ be defined by $f^{-N_{4}} \phi(\psi$, $\left.\rho, 8 L_{1}, N_{4}\right)$, where $\phi\left(\psi, \rho, 8 L_{1}, N_{4}\right)$ is given in the main lemma.

Let $N_{0} \geqq N_{1}$ be a given integer. Then, $N_{0} \geqq N_{1}>N_{3}>N_{2}$. Therefore, we have an embedding $\phi\left(j, 1 / 2,8, N_{0}\right): E^{s}(r) \times E^{u}(r) \rightarrow E^{s}\left(r_{2}\right) \times E^{u}\left(r_{2}\right)$ by the main lemma. Now, define $\phi_{0}: E^{s}(r) \times E^{u}(r) \rightarrow E^{s}(r) \times E^{u}(r)$ by $\phi_{0}$ $=f^{-N_{0}} \circ \phi\left(j, 1 / 2,8, N_{0}\right)$. 
Put $B_{0}^{u}=E^{u}(r)$ and $B_{1}^{u}=D^{u}$. Then, by our construction, (3.4) and (3.5) are satisfied. By the definition of $\phi_{0}, \phi_{1}$ and (18), we see (3.6) holds. Also, by the definition of $\phi_{0}, \phi_{1}$ and the fact $\rho<1 / 2$, (3.7) holds. (3.8) is a consequence of the definition of $\phi_{0}, \phi_{1}$, and (19). Also, (3.9) is a consequence of the definition of $\phi_{0}, \phi_{1}$ and (20).

Now, we can extend $\phi_{i}(i=0,1)$ to an embedding $\phi_{i}: E^{s}(r) \times E^{u}\left(r_{2}\right)$ $\rightarrow E^{s}(r) \times E^{u}\left(r_{2}\right)$ such that it satisfies conditions (3.1), (3.2), and $\phi_{i}\left(\partial E^{s}(r)\right.$ $\left.\times E^{u}\left(r_{2}\right)\right) \subset \partial E^{s}(r) \times E^{u}\left(r_{2}\right)$. Then, (3.3) is a consequence of the fact that $\pi^{u} \mid f^{N_{3}}\left(D^{u}\right)$ is a diffeomorphism and its image contains $E^{u}(r)$.

By the definition of $\phi_{1}$ and (14), $f^{N_{4}} \phi_{1}\left(E^{s}(r) \times B_{1}^{u}\right) \cap E^{s}(r) \times E^{u}(r)=\phi$. Also, it follows from the definition that $f^{N_{4}} \phi_{0}\left(E^{s}(r) \times B_{0}^{u}\right)=f^{-\left(N_{0}-N_{4}\right)} \phi(j, 1 / 2$, $\left.8, N_{0}\right)\left(E^{s}(r) \times B_{0}^{u}\right) \subset f^{-\left(N_{0}-N_{4}\right)}\left(E^{s}(r) \times E^{u}(r)\right) \subset E^{s}(r) \times E^{u}(r)$, since $N_{0}-N_{4} \geqq$ $N_{1}-N_{4}=N_{3}>N_{2}=N(j, 1 / 2,8)$. Therefore, $f^{N_{4}} \phi_{1}\left(E^{s}(r) \times B_{1}^{u}\right) \cap f^{N_{4}} \phi_{0}\left(E^{s}(r)\right.$ $\left.\times B_{0}^{u}\right)=\phi$. This proves (3.10).

Finally, put $k=N_{4}+L$. Then, $0 \leqq k<N_{4}+N_{3}=N_{1} \leqq N_{0}$. By (10) and our construction, $f^{k}\left(A_{1}\right) \cap f^{i}\left(A_{1}\right) \subset f^{L}(W) \cap f^{i-k+L}(W)=\phi$ for $k<i<$ $N_{0}$. Thus, $f^{k}\left(A_{1}\right) \cap f^{i}\left(A_{1}\right)=\phi$ for $k<i<N_{1}$. Also, if $0 \leqq i<k, f^{k}\left(A_{1}\right) \cap$ $f^{i}\left(A_{1}\right)=\phi$ by (9). Finally, if $0 \leqq i<N_{0}, f^{i}\left(A_{0}\right) \subset E^{s}(r) \times E^{u}(r)$. Therefore, by the fact that $f^{k}\left(A_{1}\right) \subset f^{L}(W)$ and (8), we have $f^{k}\left(A_{1}\right) \cap f^{i}\left(A_{0}\right)=\phi$ for 0 $\leqq i<N_{0}$. This completes the proof.

Let $\sum=\left\{A_{0}, A_{1}\right\}^{z}$ be a two-sided shift on two symbols $A_{0}$ and $A_{1}$. By definition, an element of $\Sigma$ is a bisequence $a=\left(a_{i}\right)_{i \in Z}$ such that $a_{i}=A_{0}$ or $A_{1}$ for each $i \in Z$, where $Z$ is the set of integers. The metric $d$ on $\Sigma$ is defined by $d(\boldsymbol{a}, \boldsymbol{b})=\sum_{i \in Z} 1 / 2^{|i|} d\left(a_{i}, b_{i}\right)$, where $\boldsymbol{a}=\left(a_{i}\right), \boldsymbol{b}=\left(b_{i}\right), d\left(a_{i}, b_{i}\right)$ $=0$ if $a_{i}=b_{i}$, and $d\left(a_{i}, b_{i}\right)=1$ if $a_{i} \neq b_{i}$. By this metric $\Sigma$ is a compact metric space.

Now, define integers $k(a, i)$ and $n(a, i)$ for $\boldsymbol{a}=\left(a_{i}\right) \in \Sigma$ as follows.

$$
\begin{aligned}
& k(\boldsymbol{a}, i)= \begin{cases}N_{0} & \text { if } a_{i}=A_{0} \\
N_{1} & \text { if } a_{i}=A_{1}\end{cases} \\
& n(\boldsymbol{a}, i)= \begin{cases}\sum_{j=0}^{i-1} k(\boldsymbol{a}, j) & \text { if } i \geqq 0 \\
-\sum_{j=-1}^{i} k(\boldsymbol{a}, j) & \text { if } i<0\end{cases}
\end{aligned}
$$

Define a subset $F^{-i}(a)$ of $M$ as follows,

$$
\boldsymbol{F}^{-i}(\boldsymbol{a})= \begin{cases}\left(f^{k(\boldsymbol{a}, 0)} \mid a_{0}\right)^{-1} \circ \cdots \circ\left(f^{k(\boldsymbol{a}, i-1)} \mid a_{i-1}\right)^{-1}\left(a_{i}\right) & \text { if } i>0 \\ a_{0} & \text { if } i=0 \\ \left.f^{k(\boldsymbol{a},-1)}\left(\cdots f^{k(\boldsymbol{a}, i+1)}\left(f^{k(a, i)}\left(a_{i}\right) \cap a_{i+1}\right) \cap \cdots\right) \cap a_{-1}\right) \cap a_{0} & \text { if } i<0 .\end{cases}
$$




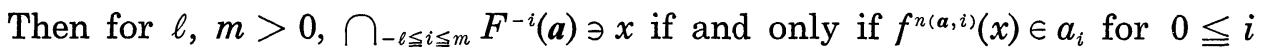
$\leqq m$, and there exist $y_{i} \in a_{i}$ with $f^{k(a, i)}\left(y_{i}\right)=y_{i+1}$ for $-\ell \leqq i<0$ and $y_{0}=x$.

Proposition 1. (a) $\bigcap_{i \in Z} F^{-i}(a)$ consists of a single point of $M$ for each $\boldsymbol{a} \in \Sigma$.

(b) A map $p: \Sigma \rightarrow M$ defined by $p(a)=\bigcap_{i \in Z} F^{-i}(a)(a \in \Sigma)$ is continuous.

(c) If there exists an integer $i \geqq 0$ such that $a_{i} \neq b_{i}$ for $a=\left(a_{i}\right), b=$ $\left(b_{i}\right) \in \Sigma$, then $p(\boldsymbol{a}) \neq p(\boldsymbol{b})$.

(d) If $N_{0}=N_{1}$, then $p \circ \sigma=f^{N_{0}} \circ p$, where $\sigma: \Sigma \rightarrow \Sigma$ is the shift map defined by $\sigma(\boldsymbol{a})=(\boldsymbol{b}), b_{i}=a_{i+1}$ for $i \in Z$.

Proof. It follows from (3.1) (3.3) that $\bigcap_{-\ell \leqq i \geqq 0} F^{-i}(\boldsymbol{a}) \neq \phi$ for any $\boldsymbol{a} \in$ $\Sigma$ and positive integer $\ell$ (cf. Kurata [2], 50-51). Let $m$ be any positive integer. For $\boldsymbol{a}=\left(a_{i}\right) \in \Sigma$, we define $\boldsymbol{b}=\left(b_{i}\right)$ by $b_{i}=a_{i+m}, i \in Z$. Then, if $x$ is a point of $\bigcap_{-(\ell+m) \leqq i \leqq 0} F^{-i}(b)\left(f^{k(a, 0)} \mid a_{0}\right)^{-1} \circ \cdots \circ\left(f^{k(a, m-1)} \mid a_{m-1}\right)^{-1}(x) \subset$ $\bigcap_{-\ell \leqq i \leqq m} F^{-i}(a)$. This implies that $\bigcap_{-\ell \leqq i \leqq m} F^{-i}(a) \neq \phi$ for any positive integers $\ell$ and $m$. Since $\bigcap_{-\ell \leqq i \leqq m} F^{-i}(a)$ is compact, $\bigcap_{i \in Z} F^{-i}(\boldsymbol{a}) \neq \phi$ for each $a \in \Sigma$.

In order to prove (a), we shall prove the following assertion (*) by induction.

(*) Let $x$ be a point of $\bigcap_{i \in Z} F^{-i}(a)$. Then, the following formula holds for any integer $\ell \geqq 0$.

$$
\bigcap_{-\ell \leqq i \leqq \ell} F^{-i}(a) \subset E^{s}\left(\pi^{s}(x) ; 2 r\left(\frac{1}{2}\right)^{\ell}\right) \times E^{u}\left(\pi^{u}(x) ; 2 r\left(\frac{1}{2}\right)^{\ell}\right),
$$

where $E^{\sigma}\left(y ; r^{\prime}\right)$ denotes the $\sigma$-dimensional disk in $E^{o}\left(r_{1}\right)$ with the center $y$ and the radius $r^{\prime}(\sigma=s, u)$.

If $\ell=0,(*)$ follows from the fact $A_{i} \subset E^{s}(r) \times E^{u}(r)(i=0,1)$. Now, assume that $(*)$ holds for some $\ell \geqq 0$.

For $\boldsymbol{a}=\left(a_{i}\right) \in \Sigma$, let $\boldsymbol{b}=\left(b_{i}\right)$ (resp. $\boldsymbol{c}=\left(c_{i}\right)$ ) be an element of $\Sigma$ given by $b_{i}=a_{i+1}\left(\right.$ resp. $\left.c_{1}=a_{i-1}\right), i \in Z$. Then,

(21) $\bigcap_{|i| \leqq \ell+1} F^{-i}(\boldsymbol{a}) \subset\left(f \mid a_{0}\right)^{-k(a, 0)}\left(\bigcap_{|i| \leqq \ell} F^{-i}(\boldsymbol{b})\right) \cap f^{k(\boldsymbol{a},-1)}\left(\bigcap_{|i| \leqq \ell} F^{-i}(\boldsymbol{c})\right)$. Let $x \in \bigcap_{i \in \boldsymbol{Z}} F^{-i}(\boldsymbol{a}), y \in \bigcap_{i \in \boldsymbol{Z}} F^{-i}(\boldsymbol{b}), w \in \bigcap_{i \in \boldsymbol{Z}} F^{-i}(\boldsymbol{c})$ be such that $f^{k(\boldsymbol{a},-1)}(w)$ $=x, f^{k(a, 0)}(x)=y$.

Define $j=0$ if $c_{0}=A_{0}$ and $j=1$ if $c_{0}=A_{1}$. Then, there is a point $w^{\prime} \in E^{s}(r)$ such that $\phi_{j}\left(w^{\prime} \times B_{j}^{u}\right) \ni w$ since $w \in c_{0}$. For $y^{\prime} \in \phi_{j}\left(w^{\prime} \times B_{j}^{u}\right) \cap$ $\phi_{j}\left(E^{s}(r) \times t^{\prime}\right)$, put $C\left(y^{\prime}\right)=\left\{z ; d\left(\pi^{s}(z), \pi^{s}\left(y^{\prime}\right)\right)<\varepsilon, z \in \phi_{j}\left(E^{s}(r) \times t^{\prime}\right)\right\}$, and for $y^{\prime \prime} \in$ $f^{k(a,-1)} \phi_{j}\left(E^{s}(r) \times t^{\prime \prime}\right), t^{\prime \prime} \in B_{j}^{n}$, put $C^{\prime}\left(y^{\prime \prime}\right)=\left\{z ; d\left(z, y^{\prime \prime}\right)<\varepsilon / 8, z \in f^{k(a,-1)} \phi_{j}\left(E^{s}(r)\right.\right.$ 
$\left.\left.\times t^{\prime \prime}\right)\right\}$, where $\varepsilon=2 r(1 / 2)^{\ell}$. Note that $\pi^{s} \phi_{j}\left(x^{\prime} \times B_{j}^{u}\right)=x^{\prime}$ and $\pi^{s}\left(y^{\prime}\right)=\pi^{s}(w)$ $=w^{\prime}$. Then, by using the inductive hypothesis, we have the following.

$$
\begin{aligned}
& \bigcap_{|i| \leqq \ell} F^{-i}(\boldsymbol{c}) \subset E^{s}\left(\pi^{s}(w) ; \varepsilon\right) \times E^{u}\left(\pi^{u}(w) ; \varepsilon\right) \subset\left(\pi^{s} \mid c_{0}\right)^{-1}\left(E^{s}\left(\pi^{s}(w) ; \varepsilon\right)\right) \\
& \quad=U\left\{C\left(y^{\prime}\right) ; y^{\prime} \in \phi_{j}\left(w^{\prime} \times B_{j}^{u}\right)\right\} .
\end{aligned}
$$

By (3.9), the following inequality holds.

$$
\begin{aligned}
& d\left(\pi^{s} \circ f^{k(a,-1)}(z), \pi^{s} \circ f^{k(a,-1)}\left(y^{\prime}\right)\right)<\frac{1}{8} d\left(\pi^{s}(z), \pi^{s}\left(y^{\prime}\right)\right), \\
& \qquad \text { for } z, y^{\prime} \in \phi_{j}\left(E^{s}(r) \times t\right) .
\end{aligned}
$$

Since $f^{k(a,-1)} \phi_{j}\left(E^{s}(r) \times t\right) \subset E^{s}(r) \times t^{\prime}$ by (3.5), it follows that

$$
d\left(\pi^{s} \circ f^{k(a,-1)}(z), \pi^{s} \circ f^{k(a,-1)}\left(y^{\prime}\right)\right)=d\left(f^{k(a,-1)}(z), f^{k(a,-1)}\left(y^{\prime}\right)\right) .
$$

Therefore, $f^{k(a,-1)}\left(C\left(y^{\prime}\right)\right) \subset C^{\prime}\left(f^{k(a,-1)}\left(y^{\prime}\right)\right)$. This and (22) imply the following.

(23) $f^{(a,-1)}\left(\bigcap_{|i| \leqq \ell} F^{-i}(c)\right) \subset \cup\left\{C^{\prime}\left(y^{\prime \prime}\right) ; y^{\prime \prime} \in f^{k(a,-1)}\left(\phi_{j}\left(w^{\prime} \times B_{j}^{u}\right)\right)\right\}$.

Define $h=0$ if $a_{0}=A_{0}$ and $h=1$ if $a_{0}=A_{1}$. Let $z^{\prime} \in B_{h}^{u}$ be a point such that $x \in \phi_{h}\left(E^{s}(r) \times z^{\prime}\right) \subset a_{0}$. For $x^{\prime} \in \phi_{h}\left(E^{s}(r) \times z^{\prime}\right)$, put

$$
B^{\prime}\left(x^{\prime}\right)=\left\{z ; d\left(\pi^{u}(z), \pi^{u} f^{k(a, 0)}\left(x^{\prime}\right)\right)<\varepsilon, z \in f^{k(a, 0)} \phi_{h}\left(t \times B_{h}^{u}\right), x^{\prime} \in \phi_{h}\left(t \times B_{h}^{u}\right)\right\}
$$

and

$$
B^{\prime}\left(x^{\prime}\right)=\left\{z ; d\left(z, x^{\prime}\right)<\varepsilon / 8, z \in \phi_{h}\left(t \times B_{h}^{u}\right), x^{\prime} \in \phi_{h}\left(t \times B_{h}^{u}\right)\right\} .
$$

Then, by using the inductive hypothesis, we have the following.

$$
\begin{aligned}
\bigcap_{|i| \leqq \ell} F^{-i}(b) & \cap f^{k(\boldsymbol{a}, 0)}\left(a_{0}\right) \subset E^{s}\left(\pi^{s}(y) ; \varepsilon\right) \times E^{u}\left(\pi^{u}(y) ; \varepsilon\right) \cap f^{k(\boldsymbol{a}, 0)}\left(a_{0}\right) \\
& \subset\left(\pi^{u}\right)^{-1} E^{u}\left(\pi^{u}(y) ; \varepsilon\right) \cap f^{k(\boldsymbol{a}, 0)} \phi_{h}\left(E^{s}(r) \times B_{h}^{u}\right) \\
& =\bigcup_{i \in E^{s}(r)}\left\{\left(\pi^{u}\right)^{-1} E^{u}\left(\pi^{u}(y) ; \varepsilon\right) \cap f^{k(\boldsymbol{a}, 0)} \phi_{h}\left(t \times B_{h}^{u}\right)\right\} .
\end{aligned}
$$

Note that $\left(\pi^{u}\right)^{-1}\left(\pi^{u}(y)\right) \cap f^{k(a, 0)}\left(a_{0}\right)=f^{k(a, 0)} \phi_{h}\left(E^{s}(r) \times z^{\prime}\right)$ and $\pi^{u} f^{k(a, 0)}\left(x^{\prime}\right)=\pi^{u}(y)$. Then, we have

$$
\bigcap_{|i| \leqq \ell} F^{-i}(b) \cap f^{k(a, 0)}\left(a_{0}\right) \subset \cup\left\{B\left(x^{\prime}\right) ; x^{\prime} \in \phi_{h}\left(E^{s}(r) \times z^{\prime}\right)\right\} .
$$

By (3.8) and the definition of $B^{\prime}\left(x^{\prime}\right)$ we have the following.

(24) $\quad\left(f^{k(a, 0)} \mid a_{0}\right)^{-1}\left(\bigcap_{|i| \leqq \ell} F^{-i}(b)\right) \subset \cup\left\{B^{\prime}\left(x^{\prime}\right) ; x^{\prime} \in \phi_{h}\left(E^{s}(r) \times z^{\prime}\right)\right\}$

Combining (21), (23) and (24), we have

(25) $\bigcap_{|i| \leqq \ell+1} F^{-i}(a) \subset\left\{\left(\cup B^{\prime}\left(x^{\prime}\right) ; x^{\prime} \in \phi_{h}\left(E^{s}(r) \times z^{\prime}\right)\right\}\right)$

$$
\cap\left(\cup\left\{C^{\prime}\left(y^{\prime \prime}\right) ; y^{\prime \prime} \in f^{k(a,-1)} \phi_{h}\left(w^{\prime} \times B_{h}^{u}\right)\right\}\right) \text {. }
$$

By definition and (3.5), if $y^{\prime \prime} \in f^{k(a,-1)} \phi_{h}\left(w^{\prime} \times B_{h}^{u}\right), d\left(z^{\prime}, y^{\prime \prime}\right)=d\left(\pi^{s}\left(z^{\prime}\right)\right.$, $\left.\pi^{s}\left(y^{\prime \prime}\right)\right)<\varepsilon / 8$ for $z^{\prime} \in C^{\prime}\left(y^{\prime \prime}\right)$ and $\pi^{u}\left(z^{\prime}\right)=\pi^{u}\left(y^{\prime \prime}\right)$. Similarly, by (3.4) and definition, if $x^{\prime} \in \phi_{h}\left(E^{s}(r) \times z^{\prime}\right), d\left(z, x^{\prime}\right)=d\left(\pi^{u}(z), \pi^{u}\left(x^{\prime}\right)\right)<\varepsilon / 8$ for $z^{\prime} \in C^{\prime}\left(y^{\prime \prime}\right)$ 
and $\pi^{s}(z)=\pi^{s}\left(x^{\prime}\right)$. These facts and (3.6), (3.7) imply that

(26) $B^{\prime}\left(x^{\prime}\right) \cap C^{\prime}\left(y^{\prime \prime}\right) \subset E^{s}\left(\pi^{s}(x) ; \varepsilon / 2\right) \times E^{u}\left(\pi^{u}(x) ; \varepsilon / 2\right)$ for any $x^{\prime} \in \phi_{h}\left(E^{s}(r)\right.$ $\left.\times z^{\prime}\right)$ and $y^{\prime \prime} \in f^{k(a,-1)} \phi_{h}\left(w^{\prime} \times B_{h}^{u}\right)$.

Therefore, (25) and (26) imply that

$$
\bigcap_{|i| \leqq \ell+1} F^{-i}(\boldsymbol{a}) \subset E^{s}\left(\pi^{s}(x) ; \frac{\varepsilon}{2}\right) \times E^{u}\left(\pi^{u}(x) ; \frac{\varepsilon}{2}\right) .
$$

Thus $(*)$ holds for $\ell+1$, and this proves $(*)$ for any $\ell \geqq 0$.

It is clear that $(*)$ implies (a).

Now, we reformulate $(*)$ as follows.

$$
\begin{gathered}
\bigcap_{-\ell \leqq i \leqq \ell} F^{-i}(\boldsymbol{a}) \subset E^{s}\left(\pi^{s} p(\boldsymbol{a}) ; 2 r\left(\frac{1}{2}\right)^{\ell}\right) \times E^{u}\left(\pi^{u} p(\boldsymbol{a}) ; 2 r\left(\frac{1}{2}\right)^{\ell}\right), \\
\text { where } p(\boldsymbol{a})=\bigcap_{i \in \boldsymbol{Z}} F^{-i}(\boldsymbol{a}), \boldsymbol{a} \in \Sigma, \text { and } \ell \geqq 0 .
\end{gathered}
$$

From this formula and the definition of the metric on $\Sigma$, continuity of $p: \Sigma \rightarrow M$ is easily proved.

By the definition of $p$, it is clear that $f^{n(a, i)}(p(a)) \in a_{i}$ for any $i \geqq 0$. Let $\boldsymbol{a}=\left(a_{i}\right), \boldsymbol{b}=\left(b_{i}\right)$ be elements of $\Sigma$ such that $a_{i} \neq b_{i}$ for some $i \geqq 0$. Then, it is clear that $f^{n(\boldsymbol{a}, i)}(p(\boldsymbol{a})) \neq f^{n(\boldsymbol{b}, i)}(p(\boldsymbol{b}))$ since $A_{0} \cap A_{1}=\phi$. Thus, $p(\boldsymbol{a})$ $\neq p(b)$.

Finally, if $N_{0}=N_{1}$, then it is clear from the definition that $p \circ \sigma=$ $f^{N_{0}} \circ \sigma$. This completes the proof.

\section{§4. Proof of the Main Theorem}

In this section, we give a proof of the main theorem.

Let $N=2 N_{1}$. Let $p$ be an integer greater than $N$. Put $N_{0}=p-N_{1}$. Then $N_{0} \geqq N_{1}$ and $p=N_{0}+N_{1}$. We apply Proposition 1 in this case.

Define $\boldsymbol{a}=\left(a_{i}\right) \in \Sigma$ by $a_{2 i}=A_{0}$ and $a_{2 i+1}=A_{1}$ for $i \in Z$. Put $x=p(\boldsymbol{a})$ $=\bigcap F^{-i}(a)$. Then, $f^{n(a, i)}(x) \in a_{i}$ for $i \geqq 0$, and there are $x_{j} \in a_{j}$ such that $f^{k(a, j)}\left(x_{j}\right)=x_{j+1}$ for $j<0$ and $x_{0}=x$. Put $y=f^{p}(x)=f^{N_{0}+N_{1}}(x)=f^{n(a, 2)}(x)$. Then, $f^{n(a, i)}(y)=f^{n(a, i)+n(a, 2)}(x)=f^{n(a, i+2)}(x)$. Hence $f^{n(a, i)}(y) \in a_{i+2}=a_{i}$ for $i \geqq$ 0 . Put $y_{j}=f^{n(a, 2)}\left(x_{j}\right)$ for $j<0$. Then, $y_{j} \in a_{j+2}=a_{j}$ and $f^{k(a, j)}\left(y_{j}\right)=f^{n(a, 2)}\left(x_{j+1}\right)$ $=y_{j+1}$ for $j<0$ and $y_{0}=y$. Therefore, $y \in \bigcap_{i \in Z} F^{-i}(a)=p(a)$ Thus, $y=$ $p(a)=x$. This proves $f^{p}(x)=x$.

Next, we shall prove that $p$ is the minimal period of $x$.

By definition, $x \in A_{0}, f^{N_{0}}(x) \in A_{1}$, and $A_{0} \cap A_{1}=\phi$. Since $z_{0} \in A_{0}$ is a fixed point of $f, x \neq z_{0}$. 
Since $N_{0} \geqq N_{1}$ and $p=N_{1}+N_{0}, N_{0} \geqq p / 2$. By the similar argument as in the proof of the main lemma, $f^{i}\left(x^{\prime}\right) \in E^{s}\left(r_{1}\right) \times E^{u}\left(r_{1}\right)$ for $0 \leqq i \leqq N_{0}$ if $x^{\prime} \in A_{0}$. Let $d$ be the minimal period of $x$.

If $d<p$, then $d \leqq p / 2$ since $d$ is a divisor of $p$. Therefore, $d \leqq N_{0}$. This implies that $f^{i}(x) \in E^{s}\left(r_{2}\right) \times E^{u}\left(r_{2}\right)$ for $0 \leqq i \leqq d$ and $f^{d}(x)=x$. But this is impossible by Hartman's theorem [1], since $x \neq z_{0}$. (We replace $r$ for a smaller value if necessary to apply Hartman's theorem.) This completes the proof of (a) of the main theorem.

Next, we prove (b) of the main theorem. In this case, we take $N_{0}=$ $N_{1}$. Then, combining Proposition 1 and Lemma 1, we have the following proposition.

Proposition 2. Under the assumption of the main theorem, we have the following properties.

(4.1) $p: \Sigma \longrightarrow M$ is a continuous map, and $p(\boldsymbol{a}) \neq p(\boldsymbol{b})$ if $a_{i} \neq b_{i}$ for some $i \geqq 0$, where $\boldsymbol{a}=\left(a_{i}\right), \boldsymbol{b}=\left(b_{i}\right) \in \Sigma$.

(4.2) $f^{N_{0}} \circ p=p \circ \sigma$.

(4.3) $A_{0} \cap A_{1}=\phi$.

(4.4) There is an integer $k\left(0 \leqq k \leqq N_{0}-1\right)$ such that $f^{k}\left(A_{1}\right) \cap f^{i}\left(A_{0}\right)=\phi$ for $0 \leqq i \leqq N_{0}-1$, and $f^{k}\left(A_{1}\right) \cap f^{i}\left(A_{1}\right)=\phi$ for $0 \leqq i \neq k \leqq N_{0}-1$.

Now, we can prove the conclusion (b) of the main theorem using Proposition 2. Our proof is similar to the one in $\mathrm{Li}$ and Yorke [3] and Marotto [4].

For $\boldsymbol{a}=\left(a_{i}\right) \in \Sigma$, let $R(\boldsymbol{a}, n)$ be the number of $a_{i}$ 's which is equal to $A_{0}$ for $0 \leqq i \leqq n$. For each $w \in(0,1)$, choose an element $\boldsymbol{a}^{w}=\left(a_{i}^{w}\right) \in \Sigma$ satisfying the following conditions.

(1) If $a_{i}^{w}=A_{0}$, then $i=k^{2}$ for some $k \in Z$.

(2) $\lim _{n \rightarrow \infty} R\left(\boldsymbol{a}^{w}, n^{2}\right) / n=w$.

Put $h=f^{N_{0}}$, and put $S_{0}=\left\{h^{k}\left(p\left(\boldsymbol{a}^{w}\right)\right) ; w \in(0,1), h \geqq 0\right\}$. Then, $h\left(S_{0}\right) \subset$ $S_{0}$ by definition. Also, by (4.2), $S_{0}=p\left\{\sigma^{k}\left(\boldsymbol{a}^{w}\right) ; w \in(0,1), k \geqq 0\right\} \supset p\left\{\boldsymbol{a}^{w} ; w \in\right.$ $(0,1)\}$. By (1), (2), and (4.1), it is clear that $p\left\{\boldsymbol{a}^{w} ; w \in(0,1)\right\}$ is uncountable. Hence $S_{0}$ is an uncountable subset of $M$.

Suppose that $x=h^{n}\left(p\left(\boldsymbol{a}^{w}\right)\right)=p\left(\sigma^{n}\left(\boldsymbol{a}^{w}\right)\right) \in S_{0}$ be a periodic point of $h$. Since $h^{\ell}(x)=h^{\ell+n}\left(p\left(\boldsymbol{a}^{w}\right)\right)=p\left(\sigma^{\ell+n}\left(\boldsymbol{a}^{w}\right)\right), h^{\ell}(x) \in a_{\ell+n}^{w}$ for any $i \geqq 0$ by the definition of $p$ and $\sigma$. Therefore, by (4.3) $a_{i}^{w}$ must be periodic in $i$ for suf- 
ficiently large $i$. But this is impossible by (1) and (2). Thus $S_{0}$ does not contain any periodic point of $h$.

Suppose that $x=h^{n}\left(p\left(\boldsymbol{a}^{w}\right)\right), y=h^{m}\left(p\left(\boldsymbol{a}^{w^{\prime}}\right)\right) \in S_{0}$, and $x \neq y$. Then, $h^{i}(x)$ $\in a_{i+n}^{w}$ and $h^{i}(y) \in a_{i+m}^{w^{\prime}}$. By (1) and (2), there exists an infinite number of $i$ 's such that $a_{n+i}^{w} \neq a_{m+i}^{w^{\prime}}$. Since $A_{0} \cap A_{1}=\phi$, and $A_{0}$ and $A_{1}$ are compact, the distance $L$ of $A_{0}$ and $A_{1}$ is positive. Therefore, there are infinite number of $i$ 's such that $d\left(h^{i}(x), h^{i}(y)\right) \geqq L>0$.

Thus, we have the following

(3) $\lim \sup _{i \rightarrow \infty} d\left(h^{i}(x), h^{i}(y)\right) \geqq L>0$ for $x, y \in S_{0}(x \neq y)$.

By a similar argument as above, we can prove that the above (3) holds for any $x \in S_{0}$ and any periodic point $y \in p(\Sigma)$ of $h$.

Let $y$ be a periodic point of $h$ in $M-p(\Sigma)$. Then, the positive orbit $\operatorname{orb}_{h}^{+}(y)=\left\{h^{i}(y) ; i \geqq 0\right\}$ of $y$ under $h$ is a finite set disjoint from the compact set $p(\Sigma)$ which contains $S_{0}$. Therefore, the following (4) holds.

(4) $\lim \sup _{i \rightarrow \infty} d\left(h^{i}(x), h^{i}(y)\right)>0$ for $x \in S_{0}$ and a periodic point $y$ of $h$.

Put $S=\left\{f^{k}(x) ; x \in S_{0}, k \geqq 0\right\}$. Then, $S \supset S_{0}$ and $f(S) \subset S$ by definition. Therefore, $S$ is an uncountable set.

If $y \in S$ is a periodic point of $f$, then there is an integer $i \geqq 0$ such that $f^{i}(y) \in S_{0}$ and $f^{i}(y)$ is a periodic point of $h=f^{N_{0}}$. But $S_{0}$ does not contain any periodic points of $h$ as stated before. Therefore, $S$ does not contain any periodic points of $f$.

Let $\tilde{\Sigma}=\left\{A_{0}, f\left(A_{0}\right), \cdots, f^{N_{0}-1}\left(A_{0}\right), A_{1}, f\left(A_{1}\right), \cdots, f^{N_{0}-1}\left(A_{1}\right)\right\}^{Z}$ be the twosided shift on $2 N_{0}$ symbols, and let $\Sigma_{1}$ be a subshift of finite type with the following transition matrix.

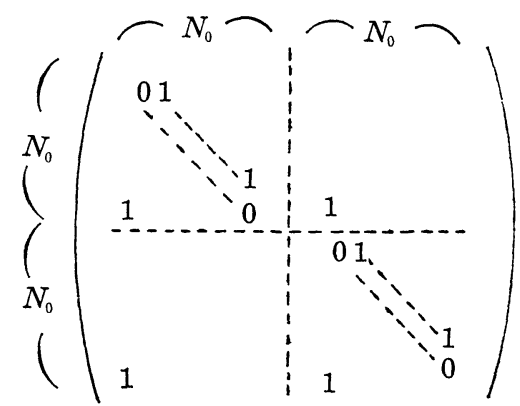

Then, by definition an element of $\Sigma_{1}$ is a bisequence of symbols consisting of the blocks of symbols of the forms $A_{0}, f\left(A_{0}\right), \cdots, f^{N_{0}-1}\left(A_{0}\right)$ and $A_{1}$, $f\left(A_{1}\right), \cdots, f^{N_{0}-1}\left(A_{1}\right)$.

Define a map $p_{1}: \Sigma_{1} \rightarrow M$ by the following manner. For $\boldsymbol{\alpha}=\left(\alpha_{i}\right) \in \Sigma_{1}$, 
$p_{1}(\alpha)$ is a point $x \in M$ satisfying the following two conditions.

(5) $f^{i}(x) \in \alpha_{i}$ for $i \geqq 0$,

(6) For each $i<0$, there is a point $y_{i} \in \alpha_{i}$ such that $f\left(y_{i-1}\right)=y_{i}$ and $y_{0}=x$.

By Proposition 1 (a), there is a unique point $x \in M$ satisfying (5) and (6). Similarly to Proposition 1, we have the following proposition.

Proposition 3. (a) $p_{1}: \Sigma_{1} \rightarrow M$ is a continuous map.

(b) If there exists an integer $i \geqq N_{0}$ such that $\alpha_{i} \neq \beta_{i}$ for $a=\left(\alpha_{i}\right), \beta$ $=\left(\beta_{i}\right) \in \Sigma_{1}$, then $p_{1}(\alpha) \neq p_{1}(\beta)$.

(c) $p_{1} \circ \sigma_{1}=f \circ p_{1}$, where $\sigma_{1}: \Sigma_{1} \rightarrow \Sigma_{1}$ is the shift map of $\Sigma_{1}$.

(d) For each $a=\left(a_{i}\right) \in \Sigma$, define $t(a)=(a)$ be an element of $\Sigma_{1}$ such that $\alpha_{N_{0}+j}=f^{j}\left(a_{i}\right)$ for $0 \leqq j \leqq N_{0}-1$, and $i \in Z$. Then, $t: \Sigma \rightarrow \Sigma_{1}$ is continuous, $p_{1} \circ t=p$, and $\sigma_{1}^{N_{0}} \circ t=t \circ \sigma$.

Now, we come back to the proof of the theorem. By Proposition 2, Proposition 3 , and the definition of $S, S=\left\{p_{1} \circ \sigma_{1}^{k} \circ t\left(\boldsymbol{a}^{w}\right) ; w \in(0,1), k \geqq 0\right\}$. By a similar argument using the properties (4.2) and (5), we can prove the conclusions (iii) and (iv) of (b) of the main theorem.

Finally, let $x=p \circ \sigma^{n}\left(\boldsymbol{a}^{w}\right), y=p \circ \sigma^{m}\left(\boldsymbol{a}^{w^{\prime}}\right) \in S_{0}$. Suppose that $m \geqq n$. Then, $m+i$ and $n+i$ cannot be squares of some integers for $i=k^{2}-n+s$, $1 \leqq s \leqq 2 k-(m-n)$. By the condition (1), we have $a_{n+i}^{w}=a_{m+i}^{w^{\prime}}$ for $i=$ $k^{2}=n+s, 1 \leqq s \leqq 2 k-(m-n)$. If we tend $k$ to infinity, we have the following.

$$
\liminf _{i \rightarrow \infty} d\left(\sigma^{n+i}\left(\boldsymbol{a}^{w}\right), \sigma^{m+1}\left(\boldsymbol{a}^{w^{\prime}}\right)\right)=0 .
$$

Since $p: \Sigma \rightarrow M$ is uniformly continuous,

$$
\liminf _{i \rightarrow \infty} d\left(p \circ \sigma_{i}\left(\boldsymbol{a}^{w}\right), p \circ \sigma^{i}\left(\boldsymbol{a}^{w^{\prime}}\right)\right)=\liminf _{i \rightarrow \infty} d\left(h^{i}(x), h^{i}(y)\right)=0 .
$$

Since $h=f^{N_{0}}$,

$$
\liminf _{i \rightarrow \infty} d\left(f^{i}(x), f^{i}(y)\right) \leqq \liminf _{i \rightarrow \infty} d\left(h^{i}(x), h^{i}(y)\right) .
$$

Thus

$$
\liminf _{i \rightarrow \infty} d\left(f^{i}(x), f^{i}(y)\right)=0 \quad \text { for } x, y \in S_{0} .
$$

This completes the proof.

Remark 6. If we replace the condition (1) by the following condition (1) ${ }^{\prime}$ if $a_{i}^{w}=A_{1}$, then $i=k^{2}$ some $k \in Z$, and if we construct the set $S$ 
similarly, then we can prove the same conclusion (b). Furthermore, (v) holds for $x, y \in S$.

\section{REFERENCES}

[1] P. Hartman, Ordinary differential equations, John Wiley and Sons, Inc. (1964), p. 245, Lemma 8.1.

[2] M. Kurata, Hartman's theorem for hyperbolic sets, Nagoya Math. J., 67 (1977), 41-52.

[ 3 ] T. Y. Li and J. A. Yorke, Period three implies chaos, Amer. Math. Monthly, 82 (1975), 985-992.

[ 4 ] F. R. Marotto, Snap-back repellers imply chaos in $\boldsymbol{R}^{n}$, J. Math. Analysis and Appl., 63 (1978), 199-223.

[ 5 ] J. Palis, On Morse-Smale dynamical systems, Topology, 8 (1969), 385-404.

[6] S. Smale, Diffeomorphisms with many periodic points, Differential and Combinatorial Topology, Princeton University Press (1965), 63-80.

Department of Mathematics

College of General Education

Nagoya University

Department of Mathematics

Nagoya Institute of Technology 